\title{
Recovery of optical properties using interstitial cylindrical diffusers as source and detector fibers
}

Timothy M. Baran 


\title{
Recovery of optical properties using interstitial cylindrical diffusers as source and detector fibers
}

\author{
Timothy M. Baran,* \\ University of Rochester Medical Center, Department of Imaging Sciences, 601 Elmwood Avenue, Box 648, Rochester, \\ New York 14642, United States
}

\begin{abstract}
We demonstrate recovery of optical properties using arrays of interstitial cylindrical diffusing fibers as sources and detectors. A single 1-cm diffuser delivered laser illumination at $665 \mathrm{~nm}$, while seven 1- and 2-cm diffusers at $1-\mathrm{cm}$ grid spacing acted as detectors. Extraction of optical properties from these measurements was based upon a diffusion model of emission and detection distributions for these diffuser fibers, informed by previous measurements of heterogeneous axial detection. Verification of the technique was performed in 15 liquid tissue-simulating phantoms consisting of deionized water, India ink as absorber, and Intralipid $20 \%$ as scatterer. For the range of optical properties tested, mean errors were $4.4 \%$ for effective attenuation coefficient, $12.6 \%$ for absorption coefficient, and $7.6 \%$ for reduced scattering coefficient. Error in recovery tended to increase with decreasing transport albedo. For therapeutic techniques involving the delivery of light to locations deep within the body, such as interstitial photodynamic and photothermal therapies, the methods described here would allow the treatment diffuser fibers also to be used as sources and detectors for recovery of optical properties. This would eliminate the need for separately inserted fibers for spectroscopy, reducing clinical complexity and improving the accuracy of treatment planning. $\odot 2016$ Society of Photo-Optical Instrumentation Engineers (SPIE) [DOI: 10.1117/1.JBO 21.7.077001]
\end{abstract}

Keywords: optical properties; cylindrical diffusing fiber; diffuser; photodynamic therapy

Paper 160324PR received May 20, 2016; accepted for publication Jul. 7, 2016; published online Jul. 22, 2016.

\section{Introduction}

Photodynamic therapy (PDT) is a treatment modality for malignant and nonmalignant diseases that relies on the combination of photosensitizer, light, and molecular oxygen to generate reactive oxygen species and cause local cell death. PDT is approved by the Food and Drug Administration (FDA) for the treatment of esophageal and endobronchial cancers, actinic keratosis, and Barrett's esophagus with high-grade dysplasia and has been used to treat a number of malignancies, often at the surface of accessible organs. ${ }^{1}$ For treatment sites that are bulky or not accessible to surface illumination, optical fibers are inserted directly into the tumor. These treatment fibers typically take the form of cylindrical diffusers, which provide roughly homogeneous emission along their distal length, and whose geometry is well suited for the treatment of large volumes. This is known as interstitial PDT (iPDT) and has been performed to treat tongue base carcinoma, ${ }^{2}$ prostate cancer, ${ }^{3}$ and cholangiocarcinoma, ${ }^{4}$ with some applications in the treatment of large skin, ${ }^{5}$ esophageal, duodenal, and colorectal tumors. ${ }^{6}$ For the FDAapproved treatment of endobronchial cancer, where either intraluminal or interstitial illumination is possible, interstitial placement of fibers is the preferred method of light delivery due to improved efficacy and reduced exposure of healthy tissue. ${ }^{7}$

Since iPDT does not allow for direct visualization of the treatment light and is often performed in proximity to healthy tissue, careful treatment planning is required to ensure that a therapeutic dose is delivered to the tumor while minimizing damage to surrounding healthy tissue. ${ }^{8}$ This dose is a combination of photosensitizer concentration (drug dose) and fluence

*Address all correspondence to: Timothy M. Baran, E-mail: timothy.baran@ rochester.edu (light dose). For a given set of tissue optical properties, absorption and scattering, this fluence distribution can be calculated or modeled for a given source geometry and strength. Optical properties can vary significantly between and within patients, so it is necessary to determine these for each individual to ensure accurate treatment planning. Without such a determination, the portion of the tumor receiving a prescribed therapeutic dose can vary widely, even for modest errors in assumed tissue absorption and scattering.

In previous iPDT studies, optical properties have been determined by a number of methods. Researchers at the University of Pennsylvania have used isotropic fibers as sources and detectors for optical property determination in iPDT of prostate cancer. ${ }^{9}$ Cylindrical diffusers also have been used as sources for optical property determination, but a separate, translatable isotropic fiber was required for detection. ${ }^{10}$ Techniques involving separate spectroscopy instruments require the insertion of additional needles into the target volume, increasing the risk and complexity of the procedure. Investigators at Lund University have replaced the treatment diffusers with flat-cleaved fibers that are also used for optical property determination, ${ }^{11}$ reducing the complexity. However, when flat-cleaved fibers are used for treatment, we have previously shown that treatment time and the number of fibers required to adequately cover the tumor volume are both increased. ${ }^{12}$ It is therefore attractive to utilize treatment diffusers for spectroscopy, to take advantage of their illumination advantages for iPDT while eliminating unnecessary needle insertions.

In this study, we demonstrate recovery of optical properties at a single wavelength from turbid samples using arrays of

$1083-3668 / 2016 / \$ 25.00$ @ 2016 SPIE 
interstitial cylindrical diffusing fibers as sources and detectors. Verification of the methods described here was performed in liquid tissue-simulating phantoms containing a scattering emulsion and ink.

\section{Materials and Methods}

\subsection{Experimental Setup}

Eight cylindrical diffusing fibers (Model 7033, Pioneer Optics Company, Bloomfield, Connecticut), four of which were $1 \mathrm{~cm}$ in length and four of which were $2 \mathrm{~cm}$ in length, were fixed in a $3 \times 3$ grid pattern at a $1-\mathrm{cm}$ separation using a brachytherapy template. This template was mounted to a post, and the distal ends of all diffusers were aligned at the same height. One of the $1-\mathrm{cm}$ diffusers, located at the corner of the grid, was connected to a 665-nm diode laser (LDX-3230-665-HHL, LDX Optronics, Inc., Maryville, Tennessee) through an inline shutter (INLINE-TTL-S, Mikropack, Ostfildern, Germany). The remaining seven diffusers, placed such that the corner of the $3 \times 3$ grid opposite the source diffuser was left empty, were connected to a thermoelectrically cooled, 16-bit spectrometer (BTC112E, B\&W Tek, Newark, Delaware) through a cascade of $1 \times 2$ optical switches $($ FSM $1 \times 2$, Piezosystem Jena, Inc., Hopedale, Massachusetts). These optical switches allowed each diffuser to be addressed sequentially by the spectrometer. A combination of 1- and 2-cm diffusers was chosen to emulate clinical conditions, in which diffusers of different lengths are often used to optimally treat tumor volumes. ${ }^{13}$ Data acquisition and instrument control were performed using a custom LabVIEW (National Instruments, Austin, Texas) interface running on a laptop computer (IBM Corporation, Armonk, New York).

\subsection{Phantom Preparation}

Measurements were made in liquid tissue-simulating phantoms consisting of deionized water, Intralipid 20\% (Baxter Healthcare Corporation, Deerfield, Illinois) as scatterer, and India ink (Higgins No. 4418, Chartpak Incorporated, Leeds, Massachusetts) as absorber. Phantoms were created at combinations of three Intralipid concentrations of $0.6 \%$ to $2.5 \%$ and five ink concentrations of $0.01 \%$ to $0.05 \%$, for a total of 15 unique phantoms. These were prepared in $1 \mathrm{~L}$ of deionized water in a 3-L container that had been painted black (Krylon Ultra-Flat Black, Sherwin-Williams Company, Solon, Ohio). Phantoms were stirred continuously during experiments using a magnetic stir bar that had also been painted black.

The India ink absorption and Intralipid scattering were characterized using a commercial spectrophotometer (Varian 50 Bio, Palo Alto, California) with a 1-cm path-length cuvette. India ink was serially diluted from 0.0125 to $0.1 \%$ in deionized water, and the optical density was measured in the spectrophotometer at each dilution. The linear relationship between measured optical density and ink concentration was used to determine the absorption coefficient, $\mu_{\mathrm{a}}$, as a function of ink concentration. Intralipid $20 \%$ was diluted to a concentration of $2 \times 10^{-7} \%$ in deionized water and measured with the spectrophotometer. Assuming negligible absorption, the measured optical density was converted to a scattering spectrum, which was found to agree well with that of van Staveren et al. ${ }^{14}$ The same bottles of India ink and Intralipid were used for all characterization and phantom preparation to minimize the effects of manufacturing heterogeneity.

\subsection{Data Collection and Correction}

Before each phantom measurement, the separations among the array of diffusers described above were measured using digital calipers at both the proximal and distal ends of the diffusing regions of the fibers. This was done to account for possible tilting or bending of the diffusers, since it could not be assumed that their axes were exactly parallel. The diffusers were then submerged to a depth of $5 \mathrm{~cm}$ in the phantom; laser illumination was delivered by one of the 1-cm diffusers and detected sequentially by each of the remaining seven diffusers. Each experiment used a fixed integration time of 5 to $2500 \mathrm{~ms}$, depending on the attenuation of the phantom, and the detected signal was quantified by integrating the detected spectrum from 663 to $667 \mathrm{~nm}$. Each measurement was repeated three times.

Spectra were background subtracted and corrected for integration time, laser power, system throughput, and fiber throughput. Background measurements were made by repeating measurements for all diffusers with the same integration time, fiber orientation, and room lighting, but with the illumination shutter closed. Laser power was measured before each experiment by inserting the source diffuser into a 6-in. calibrated integrating sphere power measurement system (Labsphere, North Sutton, New Hampshire), with each detected signal then divided by this measured power and the set integration time. System and fiber throughput were determined by connecting the $665-\mathrm{nm}$ laser to a microlens-terminated fiber whose output was measured with a thermopile detector (Model 818P-010-12, Newport Corporation, Irvine, California). Each detector diffuser was inserted into the center of the integrating sphere described above while connected to the spectrometer through the optical switch cascade. The output of the microlens-terminated fiber was used to illuminate the sphere, and the signal detected through the diffuser and system was recorded. After correction for integration time, the relationship between the known optical power striking the diffuser surface and the detected spectrum was used to determine a constant scaling factor for each fiber to correct for loss through the system. The effect of index mismatch between these calibration measurements made in air and those made in phantoms was further characterized by making measurements in five separate phantoms with known optical properties.

\subsection{Optical Property Recovery Algorithm}

Recovery of optical properties from measured, corrected data was performed in two steps: (1) determination of $\mu_{\text {eff }}$ based on fitting a theoretical model to the shape of normalized data ${ }^{15}$ and (2) separation of absorption coefficient $\left(\mu_{\mathrm{a}}\right)$ and reduced scattering coefficient $\left(\mu_{\mathrm{s}}^{\prime}\right)$ based on calibrated measures of non-normalized data. For both steps, the detected signal at a given detector $n$ was assumed to follow the expression:

$\operatorname{Det}(n)=\iiint \Phi(x, y, z) \Psi_{n}(x, y, z) \mathrm{d} x \mathrm{~d} y \mathrm{~d} z$,

where $\Phi$ is the fluence distribution for the source diffuser and $\Psi_{n}$ is the detection distribution for diffuser $n$, also known as positional importance. ${ }^{16}$ Multiple investigators have demonstrated that the fluence distribution generated by a cylindrical diffuser can be represented well as a superposition of point sources. ${ }^{17-19}$ Assuming the diffusion approximation, the source term can therefore be represented as the line integral along the diffuser axis 


$$
\Phi(x, y, z)=\frac{P}{4 \pi D} \int_{\left(x_{0}, y_{0}, z_{0}\right)}^{\left(x_{\mathrm{d}}, y_{\mathrm{d}}, z_{\mathrm{d}}\right)} \frac{e^{-\mu_{\mathrm{eff}} \sqrt{\left(x-l_{1}\right)^{2}+\left(y-l_{2}\right)^{2}+\left(z-l_{3}\right)^{2}}}}{\sqrt{\left(x-l_{1}\right)^{2}+\left(y-l_{2}\right)^{2}+\left(z-l_{3}\right)^{2}}} \mathrm{~d} l,
$$

where $P$ is the total energy per unit diffuser length delivered by the source fiber $(\mathrm{J} / \mathrm{cm}), \quad D=1 /\left[3\left(\mu_{\mathrm{a}}+\mu_{\mathrm{s}}^{\prime}\right)\right], \quad \mu_{\text {eff }}=$ $\sqrt{3 \mu_{\mathrm{a}}\left(\mu_{\mathrm{a}}+\mu_{\mathrm{s}}^{\prime}\right)}$, and $\left(x_{0}, y_{0}, z_{0}\right)$ and $\left(x_{\mathrm{d}}, y_{\mathrm{d}}, z_{\mathrm{d}}\right)$ are the coordinates of the proximal and distal ends of the source diffuser, respectively. $l$ represents the Cartesian coordinates $\left(l_{1}, l_{2}, l_{3}\right)$ along the source diffuser axis, from proximal to distal end. Again assuming the diffusion approximation, the detection distribution for diffuser $n$ can be represented as

$$
\begin{aligned}
& \Psi_{n}(x, y, z) \\
& =\frac{1}{4 \pi D} \int_{\left(x_{0, n}, y_{0, n}, z_{0, n}\right)}^{\left(x_{\mathrm{d}, n}, y_{\mathrm{d}, n}, z_{\mathrm{d}, n}\right)} \frac{e^{-\mu_{\mathrm{eff}}} \sqrt{\left(x-l_{1}\right)^{2}+\left(y-l_{2}\right)^{2}+\left(z-l_{3}\right)^{2}}}{\sqrt{\left(x-l_{1}\right)^{2}+\left(y-l_{2}\right)^{2}+\left(z-l_{3}\right)^{2}}} \xi_{n}(l) \mathrm{d} l,
\end{aligned}
$$

where $\left(x_{0, n}, y_{0, n}, z_{0, n}\right)$ and $\left(x_{\mathrm{d}, n}, y_{\mathrm{d}, n}, z_{\mathrm{d}, n}\right)$ are the coordinates of the proximal and distal ends of diffuser $n$, respectively, $l$ represents the Cartesian coordinates $\left(l_{1}, l_{2}, l_{3}\right)$ along the axis of detector diffuser $n$, from proximal to distal end, and $\xi_{n}(l)$ is the diffuser detection profile. These detection profiles represent the probability that a photon striking the diffusive region at a given axial position will be coupled into the fiber core and ultimately reach the spectrometer. We have previously shown that while diffuser emission is roughly homogeneous, this detection profile is highly heterogeneous. ${ }^{19}$ Detection profiles for the diffusers used in this study were characterized in a previous study and shown to compare favorably to Monte Carlo simulations. ${ }^{20}$ Briefly, an isotropic probe was coupled to a laser source at $665 \mathrm{~nm}$ and placed $\sim 1 \mathrm{~mm}$ from the diffuser surface, while the diffuser was connected to a spectrometer. This probe was translated along the diffuser axis in $0.5 \mathrm{~mm}$ intervals, with a spectrum captured at each interval. These spectra were then integrated from 663 to $667 \mathrm{~nm}$ and normalized to the mean integrated signal to generate diffuser detection profiles.

For determination of $\mu_{\text {eff }}$, the detected signal at each diffuser was normalized to the maximum signal detected by any one diffuser and fit with Eqs. (1)-(3) using constrained, nonlinear optimization with $\mu_{\text {eff }}$ constrained to be positive. Since the data are normalized in this step, only the shape of the $\operatorname{Det}(n)$ versus $n$ curve can be fit. This is sufficient to recover the value of $\mu_{\text {eff }}$, but determination of $\mu_{\mathrm{a}}$ and $\mu_{\mathrm{s}}^{\prime}$ requires a calibrated measurement of absolute signal. To separate absorption and scattering, $\mu_{\text {eff }}$ was set to the value extracted in the first step of the algorithm, and detected fluence was calculated using Eqs. (1)-(3) and an assumed value of $D$. This assumed value was then multiplied out, and $D$ was determined from the mean ratio of the measured, calibrated detection to the calculated detection at the three diffusers with the greatest signal. Since this is a ratiometric technique, division of nonzero measured signals at the largest separation from the source fiber by calculated values close to zero resulted in these remote fibers having an erroneously high contribution to the fit. This is why only the three detectors with greatest signal were included in this step. Using the fitted values of $\mu_{\text {eff }}$ and $D, \mu_{\mathrm{a}}$ and $\mu_{\mathrm{s}}^{\prime}$ could be calculated. All computation and fitting were performed using MATLAB (The Mathworks, Inc., Natick, Massachusetts).

The number of detectors required for accurate recovery of $\mu_{\text {eff }}$ was examined by using the first phase of the fitting algorithm described above, while presenting progressively fewer $\operatorname{Det}(n)$ to the fitting routine. This was done using the same phantom data collected as described above and discarding certain $\operatorname{Det}(n)$ in the fitting software. Detected signals were removed from most remote to closest in relation to the proximal end of the source diffuser. In cases in which two detector diffusers were equidistant, the next detector to be removed was chosen at random. Error versus number of detectors was quantified by pooling all recovered $\mu_{\text {eff }}$ for a particular number of detectors used and computing the mean and standard deviation of the percent error compared to known values.

\section{Results}

Normalized, detected fluence as a function of radial sourcedetector separation at the proximal diffuser ends and the resulting fit using Eqs. (1)-(3) are shown in Fig. 1(a) for a single phantom with $\mu_{\text {eff }}=4.4 \mathrm{~cm}^{-1}$. Note that four of the detection diffusers were $2 \mathrm{~cm}$ in length and three were $1 \mathrm{~cm}$ in length, and diffuser axes were not necessarily parallel. So the detected
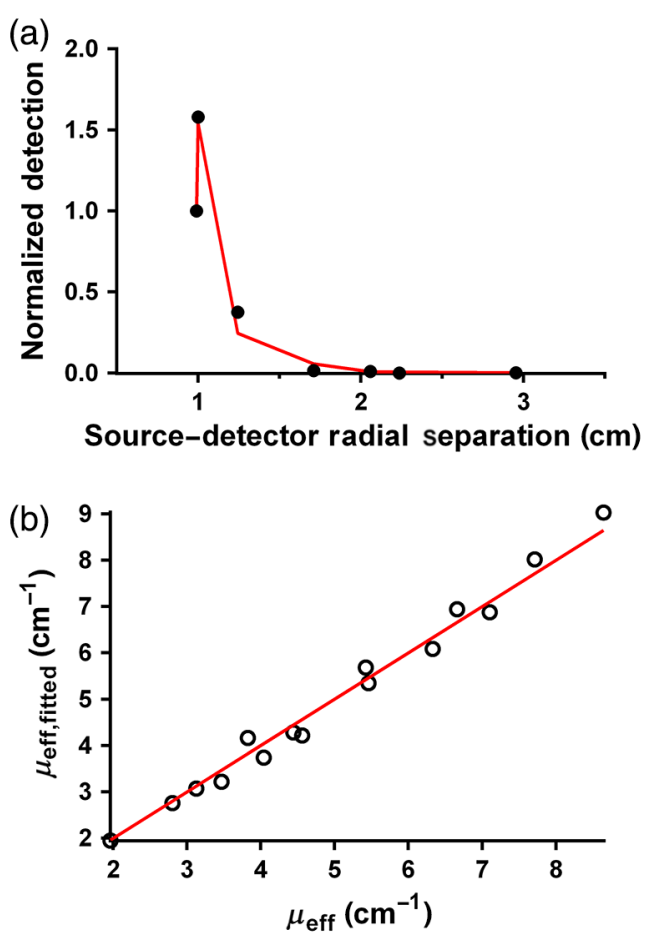

Fig. 1 (a) Measured, normalized detection versus source-detector radial separation at the proximal diffuser ends for measurements made in a single phantom with $\mu_{\text {eff }}=4.4 \mathrm{~cm}^{-1}$ (filled circles). The solid line represents the calculated detection pattern for the fitted value of $\mu_{\mathrm{eff}}=4.3 \mathrm{~cm}^{-1}$. Note that four of the detectors were $2 \mathrm{~cm}$ in length while three were $1 \mathrm{~cm}$, and diffuser axes were not necessarily parallel. So the detected signal does not fall off smoothly with radial source-detector separation at the proximal diffuser ends. (b) Fitted $\mu_{\text {eff }}$ values (open circles) determined for measurements made in 15 liquid phantoms show good agreement with known phantom optical properties. Data points shown are mean values for three repeated measurements in each phantom, with errors bars representing standard deviation being smaller than the data points in all cases. The solid line represents perfect agreement. 

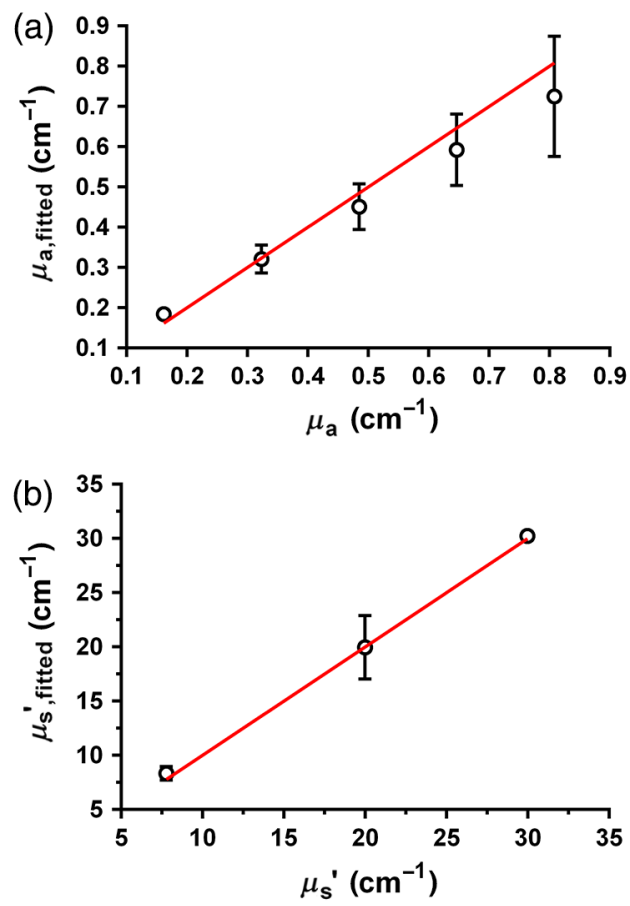

Fig. 2 Recovery of (a) $\mu_{\mathrm{a}}$ and (b) $\mu_{\mathrm{s}}^{\prime}$ from measurements made in liquid phantoms. Each data point in (a) corresponds to the mean value determined from phantom measurements, in which $\mu_{\mathrm{a}}$ was fixed at one of five values, but $\mu_{\mathrm{s}}^{\prime}$ varied over the three values shown in (b). Each data point in (b) represents the mean $\mu_{\mathrm{s}}^{\prime}$ value found for phantoms with equal reduced scattering coefficient, but with the varying values of $\mu_{\mathrm{a}}$ shown in (a). Error bars in both cases are standard deviation, with solid lines showing perfect agreement.

signal does not fall off smoothly with radial source-detector separation at the proximal diffuser ends due to the increased surface area of detection for the 2-cm diffusers and varying source-detector separation at distal diffuser ends due to diffuser axes not being parallel. For the curve shown in Fig. 1(a), the extracted value of $\mu_{\text {eff }}$ was $4.3 \mathrm{~cm}^{-1}$. The extracted values of $\mu_{\text {eff }}$ for all phantom measurements are shown in Fig. 1(b), with the solid red line representing perfect agreement. The mean error in recovery of $\mu_{\text {eff }}$ was $4.4 \%$, with a maximum error of $8.9 \%$.

Extracted $\mu_{\mathrm{a}}$ and $\mu_{\mathrm{s}}^{\prime}$ values are shown in Fig. 2. For the recovered values of $\mu_{\mathrm{a}}$ shown in Fig. 2(a), each data point corresponds to the mean value determined from phantom measurements, in which the absorption coefficient was fixed at one of five values, but the amount of scatter varied over three concentrations. Similarly, each data point in Fig. 2(b) represents the mean $\mu_{\mathrm{s}}^{\prime}$ value found for phantoms with equal reduced scattering coefficient but varying absorption. Mean error in the recovery of absorption coefficient was $12.6 \%$, with a maximum error of $23.9 \%$. Mean error in the determination of reduced scattering coefficient was $7.6 \%$, with a maximum error of $20.7 \%$.

For recovered optical properties, the error tended to increase with decreasing transport albedo $\left[a^{\prime}=\mu_{\mathrm{s}}^{\prime} /\left(\mu_{\mathrm{a}}+\mu_{\mathrm{s}}^{\prime}\right)\right]$, as shown in Fig. 3. Error in recovery was negatively correlated with $a^{\prime}$ for both $\mu_{\mathrm{a}}$ (Pearson $r=-0.59$ ) and $\mu_{\mathrm{s}}^{\prime}$ (Pearson $r=-0.33$ ).

Error in the recovery of $\mu_{\text {eff }}$ increased with a decreasing number of detected signals used in the fitting algorithm, as shown in Fig. 4. When at least three detectors were included in fitting, the mean error did not exceed $6.5 \%$, compared to a mean error of $4.4 \%$ when all seven detectors were used. For the case of only

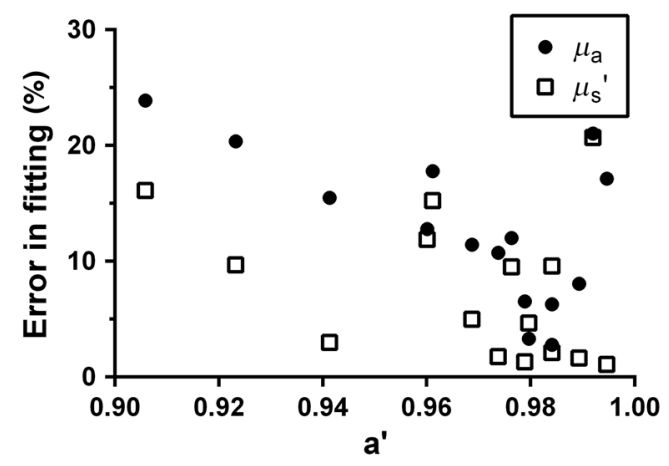

Fig. 3 The relationship between error in recovery of $\mu_{\mathrm{a}}$ (filled circles) and $\mu_{\mathrm{s}}^{\prime}$ (open squares) and the transport albedo $\left(a^{\prime}\right)$ shows a trend of increasing error with decreasing transport albedo. Error in recovery was negatively correlated with $a^{\prime}$ for both $\mu_{\mathrm{a}}(r=-0.59)$ and $\mu_{\mathrm{s}}^{\prime}$ $(r=-0.33)$.

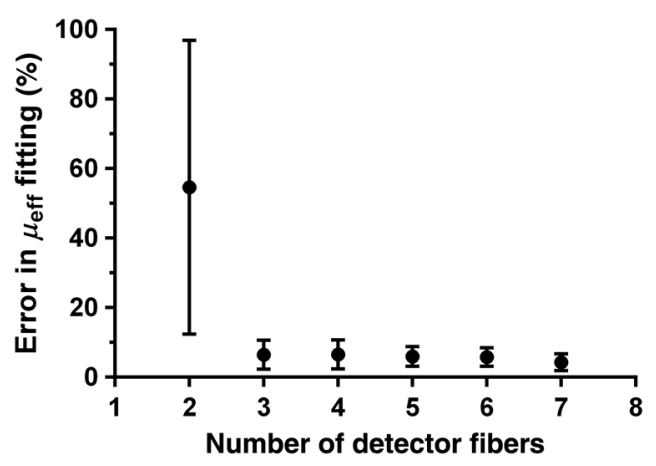

Fig. 4 The error in recovery of $\mu_{\text {eff }}$ increases with a decreasing number of detector fibers utilized. Data points are means of results from all experiments, with the algorithm run for a given number of detector fibers, while error bars are standard deviation.

two detectors, the mean error increased markedly to $54.6 \%$, perhaps representing a fundamental limit for this technique.

\section{Discussion}

A number of research groups have examined the recovery of optical properties from interstitial measurements based on steady-state reflectance spectroscopy. Chin et al. ${ }^{21}$ demonstrated accuracy to within $\sim 20 \%$ for measurements made in phantoms containing Intralipid and Naphthol Green using relative steady-state reflectance measurements. Dimofte et al. ${ }^{10}$ showed recovery of optical properties using diffusers as sources, but with isotropic point detectors. When the value of $\mu_{\mathrm{s}}^{\prime}$ was not fixed $a$ priori, the standard deviation for determining $\mu_{\mathrm{a}}$ in a homogeneous phantom was $18 \%$. In our previous work using a custom, interstitial optical probe, we were able to achieve a mean error of $9 \%$ in the recovery of absorption and $19 \%$ in the recovery of scattering. ${ }^{22}$ The mean errors of $12.6 \%$ and $7.6 \%$ in determination of $\mu_{\mathrm{a}}$ and $\mu_{\mathrm{s}}^{\prime}$, respectively, that we demonstrate here compare favorably to these results, without requiring separate fibers for spectroscopy.

As shown in Fig. 3, the error in the recovery of optical properties increases with decreasing transport albedo. This is expected, as the diffusion approximation utilized here is valid only for values of $a^{\prime}$ that are close to 1 , with a common cutoff being $a^{\prime}>0.98 .^{23}$ If phantoms for which the transport albedo 
was $<0.98$ are excluded, the mean error in recovery improves to $11.1 \%$ for $\mu_{\mathrm{a}}$ and $7.0 \%$ for $\mu_{\mathrm{s}}^{\prime}$. While this is not a large improvement in the accuracy of the algorithm, it does motivate a possible examination of higher order approximations to the Boltzmann transport equation for representation of diffusers. One such model is the $\mathrm{P}_{3}$ approximation, which expresses the terms in the Boltzmann equation using a third-order expansion in spherical harmonics. We have previously demonstrated the utility of this approximation in the context of reflectance ${ }^{24}$ and interstitial fluorescence spectroscopy. ${ }^{16}$ Applying this technique to the current problem would require representing the diffuser emission and detection as a superposition of $\mathrm{P}_{3}$ point sources. While this could improve accuracy, the computational complexity would also be increased. Even with the simpler diffusion model described here, the optical property recovery algorithm requires $\sim 8$ min to determine $\mu_{\mathrm{a}}$ and $\mu_{\mathrm{s}}^{\prime}$ for a single set of measurements on a modern desktop computer. Using the $\mathrm{P}_{3}$ approximation would further slow this process, possibly precluding the technique from use in a clinical setting. As an example, for the simple case of steady-state, spatially resolved, and surface-contact diffuse reflectance, the use of the $\mathrm{P}_{3}$ approximation results in $\sim 50 \%$ longer runtime compared to the diffusion approximation. Additionally, the straightforward determination of $D$ from the ratio of calculated and measured data would not be possible due to the multiple exponential terms in the $\mathrm{P}_{3}$ point-source representation. ${ }^{23}$ Development of an algorithm utilizing the $\mathrm{P}_{3}$ approximation would require further investigation to determine its feasibility and potential improvement in accuracy.

The impact of attenuation by the other diffusers was ignored in Eqs. (1)-(3). This would be the case in which light reaching a remote detection fiber has passed through another diffuser. We have neglected this for two reasons, the first being that absorption is negligible in the diffusive regions of these fibers. So the attenuation due to diffuser absorption is expected to be low. Scattering through intervening diffusers is also a potential issue. For the diffusers used in this study, we have previously shown that the scattering coefficient within the diffusive region is small for all but the most distal regions. ${ }^{20}$ Using the BeerLambert law, this results in $95 \%$ of light remaining unscattered during transmission through the diffusive region for all but the most distal $5 \mathrm{~mm}$. Since scattering within the diffuser is assumed to be isotropic due to the scatterer size, some amount of this scattered light will continue propagating in a similar direction to the incident light. Upon re-entering the surrounding turbid medium, these photons will again experience multiple scattering, resulting in the change in propagation direction being effectively negated over short length scales in the sample. Therefore, we expect the effects of diffuser scattering to be minimal.

The accuracy of $\mu_{\mathrm{a}}$ and $\mu_{\mathrm{s}}^{\prime}$ separation described here was based on calibration of the absolute detected signal in phantoms with the same index of refraction as those used for optical property extraction experiments. This technique would therefore not be as accurate in the determination of optical properties for a sample of unknown refractive index. Further characterization would be required to determine the effects of index on the emission and detection behavior of the diffusers, as has been performed for isotropic dosimetry probes. ${ }^{25}$ It is anticipated that the recovery of $\mu_{\text {eff }}$ would be more robust to changes in refractive index, as this determination is made based on relative measures of detected fluence, rather than an absolute signal.

The goal of the optical property determination described here is to allow for the creation of accurate, individualized treatment

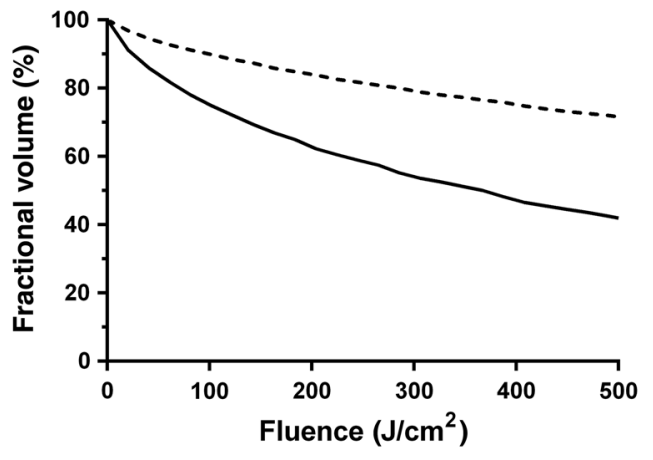

Fig. 5 DVHs showing the percentage of a tumor volume derived from CT scans of a head and neck cancer patient who received a particular simulated fluence (light dose). The solid line represents a scenario in which the treatment plan was made assuming an erroneous $\mu_{\mathrm{a}}=$ $0.2 \mathrm{~cm}^{-1}$, while the dashed line represents a scenario in which the treatment plan was made assuming the correct $\mu_{\mathrm{a}}=0.4 \mathrm{~cm}^{-1}$.

plans for iPDT. It is hypothesized that this treatment planning could improve tumor response and reduce damage to surrounding healthy tissue. This has been undertaken by a number of research groups, largely in the context of prostate cancer. ${ }^{8,13}$ The methods described here could be readily integrated with treatment planning software to allow for customized therapy. We have previously demonstrated treatment planning software for iPDT that can optimize diffuser length, placement, and total energy delivered based on patient CT images. ${ }^{12}$ The impact of measured optical properties can be evaluated using the dose volume histogram (DVH) generated by this treatment planning software, which shows what portion of the tumor volume received a particular dose. This was performed for two scenarios based on the same CT scans of a head and neck cancer patient, with a difference of $0.2 \mathrm{~cm}^{-1}$ between the assumed and measured $\mu_{\mathrm{a}}$. For the erroneous assumed absorption, this resulted in a plan utilizing eight cylindrical diffusing fibers each delivering 400 to $1000 \mathrm{~J} / \mathrm{cm}$. The simulated DVH resulting from this is shown as the solid line in Fig. 5. In a clinical environment, this difference in tissue absorption could not be accounted for without a pretreatment measurement of optical properties. When the treatment plan was updated using the correct value of $\mu_{\mathrm{a}}$, representing the case in which a determination of optical properties was performed pretreatment, the portion of the tumor receiving the desired light dose was improved. In this case, an incorrect assumption of absorption coefficient in treatment planning led to the portion of the tumor receiving the desired light dose being reduced from $90 \%$ to $76 \%$.

The methods described here are vulnerable to a number of clinical considerations that could reduce accuracy. As seen in Eqs. (2) and (3), this algorithm relies upon knowledge of the positions of the proximal and distal ends of each diffuser and assumes that the diffusers are straight between these bounds. For the experiments described here, direct measurement of these coordinates was possible. In a clinical scenario, this would not be possible. Fortunately, many of the diffusers utilized, such as the Model RD (Medlight SA, Ecublens, Switzerland), are offered with radiopaque bands at both ends of the diffusive region. This would allow for accurate determination of diffuser positioning through the use of a CT scanner in the procedure room, which is present in our hospital and in many other centers that perform iPDT. These diffusers are often contained in a 
clear, rigid catheter during treatment, which would ensure that linearity of the diffuser axis is maintained.

Another clinical concern is the presence of heterogeneities within the tumor volume, particularly those induced by bleeding. When the catheters containing the diffusers are inserted into the tumor volume, this could result in blood pooling around the source and detector fibers. Since the model assumes homogeneous optical properties, this localized increase in absorption would be seen only as decreased detection by other fibers. This would result in the extracted $\mu_{\mathrm{a}}$ being larger than expected for all regions, as the effect of this bleeding is averaged over the entire path between source and detector. Additionally, tissue optical properties can be heterogeneous, meaning that measurements made over a large array of diffusers will provide results that are a superposition of the sampled absorption and scattering values. Performing measurements with a smaller number of diffusers would shrink the volume interrogated by spectroscopy and improve sensitivity to spatially heterogeneous optical properties. As we have shown here, three detection fibers are required to achieve a mean error of $<6.5 \%$ in the determination of $\mu_{\text {eff }}$. So for a clinical scenario in which more than three fibers are utilized, subsets of these fibers could be addressed in groups of four (one source and three detectors) to coarsely sample these heterogeneities.

For iPDT applications, the number of diffusers utilized can vary widely based on the tumor volume and patient anatomy. In the treatment of large tumors of the head and neck, the number of simultaneous treatment fibers is often four. ${ }^{26,27}$ Similarly for iPDT of the prostate, generally between 4 and 12 fibers have been employed for therapeutic illumination, ${ }^{13,28}$ with 4 or more separate detector fibers inserted. Although similar accuracy was shown for the technique described here with fewer than seven detectors used, there was an increase in error with decreasing number of detectors. So for larger tumor volumes in which a greater number of diffusers are used, more of these could act as detectors for spectroscopy, potentially increasing the accuracy of optical property extraction. As discussed above, this would result in a larger area being sampled, reducing the sensitivity to heterogeneities. A trade-off between accuracy and localization would therefore be required in clinical applications.

The techniques described here will not allow for alteration of fiber placement after spectroscopy measurements, but will allow for modification of the illumination duration at each treatment diffuser. For the case described above and illustrated in Fig. 5, the illumination duration would need to be increased by approximately a factor of 4 to account for the measured increase in absorption, resulting in each fiber delivering 1600 to $4400 \mathrm{~J} / \mathrm{cm}$. In this way, we expect that the methods described here could lead to improved tumor coverage during iPDT and therefore improved outcome. Since this technique does not require the insertion of additional fibers, spectroscopic measurements could also be performed during treatment illumination by temporarily decoupling specific fibers from the treatment laser and routing them to a spectrometer via optical switching. This would allow for real-time determination of tissue optical properties in the volume being treated, which could be used to detect changes in hemoglobin oxygen saturation and photosensitizer concentration for photosensitizers with sufficient extinction at the treatment wavelength. This real-time measurement could be coupled to dynamic treatment planning software to provide safer and more accurate therapy to patients.

\section{Acknowledgments}

This work was supported by the National Institutes of Health under Grants Nos. CA68409 and CA55791 awarded by the National Cancer Institute. The author would like to thank Dr. Thomas Foster for his detailed review and discussion of this paper.

\section{References}

1. P. Agostinis et al., "Photodynamic therapy of cancer: an update," $C A$ : Cancer J. Clin. 61, 250-281 (2011).

2. W. Jerjes et al., "Photodynamic therapy: the minimally invasive surgical intervention for advanced and/or recurrent tongue base carcinoma," Lasers Surg. Med. 43, 283-292 (2011).

3. H. Patel et al., "Motexafin lutetium-photodynamic therapy of prostate cancer: short- and long-term effects on prostate-specific antigen," Clin. Cancer Res. 14, 4869-4876 (2008).

4. M.-A. Ortner, "Photodynamic therapy for cholangiocarcinoma," Lasers Surg. Med. 43, 776-780 (2011).

5. A. Johansson et al., "In vivo measurement of parameters of dosimetric importance during interstitial photodynamic therapy of thick skin tumors," J. Biomed. Opt. 11, 034029 (2006).

6. J. Regula et al., "Photosensitisation and photodynamic therapy of oesophageal, duodenal, and colorectal tumours using 5 aminolaevulinic acid induced protoporphyrin IX: a pilot study," Gut 36, 67-75 (1995).

7. Pinnacle Biologics, Inc., Photofrin (Package Insert), Pinnacle Biologics, Inc., Bannockburn, Illinois (2011).

8. S. R. H. Davidson et al., "Treatment planning and dose analysis for interstitial photodynamic therapy of prostate cancer," Phys. Med. Biol. 54, 2293-2313 (2009).

9. T. C. Zhu, J. C. Finlay, and S. M. Hahn, "Determination of the distribution of light, optical properties, drug concentration, and tissue oxygenation in-vivo in human prostate during motexafin lutetium-mediated photodynamic therapy," J. Photochem. Photobiol. B 79, 231-241 (2005).

10. A. Dimofte et al., "Determination of optical properties in heterogeneous turbid media using a cylindrical diffusing fiber," Phys. Med. Biol. 57, 6025-6046 (2012).

11. J. Swartling et al., "System for interstitial photodynamic therapy with online dosimetry: first clinical experiences of prostate cancer," J. Biomed. Opt. 15, 058003 (2010).

12. T. M. Baran and T. H. Foster, "Comparison of flat cleaved and cylindrical diffusing fibers as treatment sources for interstitial photodynamic therapy," Med. Phys. 41, 022701 (2014).

13. M. D. Altschuler et al., "Optimized interstitial PDT prostate treatment planning with the Cimmino feasibility algorithm," Med. Phys. 32, 3524-3536 (2005).

14. H. J. van Staveren et al., "Light scattering in Intralipid-10\% in the wavelength range of 400-1100 nm," Appl. Opt. 30, 4507-4514 (1991).

15. T. M. Baran and T. H. Foster, "Use of cylindrical diffusing fibers as detectors for interstitial tissue spectroscopy," Proc. SPIE 9308, 93080J (2015).

16. T. M. Baran and T. H. Foster, "Recovery of intrinsic fluorescence from single-point interstitial measurements for quantification of doxorubicin concentration," Lasers Surg. Med. 45, 542-550 (2013).

17. L. H. Murrer, H. P. Marijnissen, and W. M. Star, "Monte Carlo simulations for endobronchial photodynamic therapy: the influence of variations in optical and geometrical properties and of realistic and eccentric light sources," Lasers Surg. Med. 22, 193-206 (1998).

18. B. Farina et al., "Monte Carlo simulation of light fluence in tissue in a cylindrical diffusing fibre geometry," Phys. Med. Biol. 44, 1-11 (1999).

19. T. M. Baran and T. H. Foster, "New Monte Carlo model of cylindrical diffusing fibers illustrates axially heterogeneous fluorescence detection: simulation and experimental validation," J. Biomed. Opt. 16, 085003 (2011).

20. T. M. Baran, "Cylindrical diffuser axial detection profile is dependent on fiber design," J. Biomed. Opt. 20, 040502 (2015).

21. L. C. L. Chin et al., "Determination of the optical properties of turbid media using relative interstitial radiance measurements: Monte Carlo study, experimental validation, and sensitivity analysis," J. Biomed. Opt. 12, 064027 (2007). 
22. T. M. Baran, M. C. Fenn, and T. H. Foster, "Determination of optical properties by interstitial white light spectroscopy using a custom fiber optic probe," J. Biomed. Opt. 18, 107007 (2013).

23. E. L. Hull and T. H. Foster, "Steady-state reflectance spectroscopy in the $\mathrm{P}_{3}$ approximation," J. Opt. Soc. Am. A 18, 584-599 (2001).

24. J. C. Finlay and T. H. Foster, "Hemoglobin oxygen saturations in phantoms and in vivo from measurements of steady-state diffuse reflectance at a single, short source-detector separation," Med. Phys. 31, 19491959 (2004).

25. J. P. A. Marijnissen and W. M. Star, "Calibration of isotropic light dosimetry probes based on scattering bulbs in clear media," Phys. Med. Biol. 41, 1191-1208 (1996).

26. P. Lou et al., "Interstitial photodynamic therapy as salvage treatment for recurrent head and neck cancer," Br. J. Cancer 91, 441-446 (2004).
27. H. R. Jäger et al., "MR imaging-guided interstitial photodynamic laser therapy for advanced head and neck tumors," AJNR Am. J. Neuroradiol. 26, 1193-1200 (2005).

28. C. M. Moore et al., "Photodynamic therapy using meso tetra hydroxy phenyl chlorin (mTHPC) in early prostate cancer," Lasers Surg. Med. 38, 356-363 (2006).

Timothy Baran is a research assistant professor in the Department of Imaging Sciences at the University of Rochester Medical Center. He received his BS degree in electrical, computer and systems engineering from Rensselaer Polytechnic Institute and his PhD in optics from the University of Rochester. His research interests include photodynamic therapy, tissue optics, high performance computing, and medical imaging. 